\title{
Quantum information processing by NMR using a 5-qubit system formed by dipolar coupled spins in an oriented molecule
}

\author{
Ranabir Das ${ }^{\mathrm{a}}$, Rangeet Bhattacharyya ${ }^{\mathrm{a}}$, Anil Kumar ${ }^{\mathrm{a}, \mathrm{b}, *, 1}$ \\ a Department of Physics, Indian Institute of Science, Bangalore, India \\ ${ }^{\mathrm{b}}$ Sophisticated Instruments Facility, Indian Institute of Science, Bangalore, India
}

\begin{abstract}
Quantum information processing by NMR with small number of qubits is well established. Scaling to higher number of qubits is hindered by two major requirements (i) mutual coupling among qubits and (ii) qubit addressability. It has been demonstrated that mutual coupling can be increased by using residual dipolar couplings among spins by orienting the spin system in a liquid crystalline matrix. In such a case, the heteronuclear spins are weakly coupled, but the homonuclear spins often become strongly coupled. In such circumstances, the strongly coupled spins, which yield second order spectra, can no longer be individually treated as qubits. However, it has been demonstrated elsewhere, that the $2^{N}$ energy levels of a strongly coupled $N$ spin-1/2 system can be treated as an $N$-qubit system. For this purpose the various transitions have to be identified to well defined energy levels. This paper consists of two parts. In the first part, the energy level diagram of a heteronuclear 5-spin system is obtained by using a newly developed heteronuclear z-cosy (HET-Z-COSY) experiment. In the second part, implementation of logic gates, preparation of pseudopure states, creation of entanglement, and entanglement transfer is demonstrated, validating the use of such systems for quantum information processing.
\end{abstract}

Keywords: Quantum information processing; NMR; Liquid crystal

\section{Introduction}

Theoretically, future quantum computers can simulate physical systems and solve certain problems more efficiently than classical computers [1-5]. This possibility has excited a number of research groups to strive for experimental realization of quantum computers. Apart from trapped ions and cavity-QED systems, nuclear magnetic resonance (NMR) has proved as a suitable physical system for quantum information processing (QIP) [6,7]. In fact, NMR has emerged as the most successful of all systems, demonstrating several quantum

\footnotetext{
${ }^{*}$ Corresponding author. Fax: +918023601550.

E-mail address: anilnmr@physics.iisc.ernet.in (A. Kumar).

${ }^{1}$ DAE-BRNS Senior Scientist.
}

algorithms including Shor's factorization algorithm [814]. However, scalability is one of the hurdles that NMR-QIP faces today.

Originally, it was conceived that liquid-state NMR will use as qubits, spin-1/2 nuclei having different Larmor frequencies and weakly coupled to each other by indirect $J$-couplings [15-17]. Since $J$-couplings mediate through covalent bonds, they become vanishingly small beyond 4-5 bonds. Such systems are thus difficult to scale beyond 7-8 qubits. Attempts are going on to explore other couplings such as the direct dipolar couplings between spin-1/2 nuclei, which are larger in magnitude, depend on the inter-nuclear distances, and not on the number of intermediate covalent bonds. However, in liquids, molecules undergo fast isotropic reorientations, thereby averaging out all dipolar interactions (homonuclear, 
heteronuclear, intermolecular, and intra molecular). In solids, the molecules are static, and all the dipolar interactions are retained yielding broad spectra, rendering such systems unsuitable for quantum information processing. However, when molecules are dissolved in liquid crystal solvents, they have sufficient translational motion, but get partially oriented and have anisotropic rotational motion. In such cases, while the intermolecular dipolar interactions are averaged to zero, the intra-molecular dipolar interactions survive which are scaled down by several fold. Such systems yield well resolved spectra with finite number of sharp lines [18]. In such cases the spins have considerable dipolar couplings and it has been demonstrated that they can be used for quantum information processing [19-23].

However, in cases where the differences in Larmor frequencies or chemical shifts are not large compared to the dipolar couplings, the spins become strongly coupled. The eigenstates of the system are not products of the eigenstates of various spins, but linear combinations of the product states, and spins can no longer be identified as a qubits [24]. Still, it has been demonstrated that the $2^{N}$ energy levels of the $N$ spin-1/2 system can be treated as $2^{N}$ states of a $N$-qubit system, and can be used for quantum information processing [25]. Quantum Information processing has already been demonstrated in homonuclear 2,3 and 4-spin systems oriented in liquid crystal matrices [25]. In the homonuclear systems, it was found that if the spins are in symmetric chemical environment, the eigenstates break up into symmetry groups, leading to reduced number of qubits and other complications with degenerate transitions from different symmetry manifolds $[21,23]$. It is therefore desirable that the homonuclear spins are in non-symmetric chemical environment. Furthermore, it is advantageous to include heteronuclear spins in the system. Here, we extend our earlier work by including a heteronuclear spin with four homonuclear spins in non-equivalent chemical environment, all dipolar coupled to each other. We use the molecule 1-chloro2-fluorobenzene (dissolved in liquid crystal solvent ZLI-1132), which yields a 5-qubit system in which the four protons are strongly coupled to each other, but weakly coupled to the fluorine. The Hamiltonian for the dipolar interaction among the strongly coupled protons $\left(\mathbf{I}_{\mathbf{i}}\right)$ is,

$H_{D}=2 \pi \sum_{i, j(i<j)} D_{i j}^{\text {ori }}\left(3 I_{i z} I_{j z}-\mathbf{I}_{\mathbf{i}} \cdot \mathbf{I}_{\mathbf{j}}\right)$,

where $D_{i j}^{\mathrm{ori}}=\frac{\gamma_{i} \gamma_{j} \hbar}{4 \pi r_{i j}^{3}} \Omega_{i j}$ is the scaled dipolar interaction, scaled by the order parameter $\Omega_{i j}[18]$. The Hamiltonian for dipolar interaction between the protons and the fluorine $(\mathbf{S})$ is

$H_{D}=2 \pi \sum_{i} D_{I_{i} S}^{\mathrm{ori}} I_{z i} S_{z}$,

The full Hamiltonian of the system including Zeeman, dipolar, and $J$-coupling terms, is given by:

$$
\begin{aligned}
H= & H_{Z}+H_{D}+H_{J} \\
= & \sum_{i} \omega_{I_{i}} I_{z i}+\omega_{s} S_{z}+2 \pi \sum_{i, j(i<j)} D_{i j}^{\mathrm{ori}}\left(3 I_{i z} I_{j z}-\mathbf{I}_{\mathbf{i}} \cdot \mathbf{I}_{\mathbf{j}}\right) \\
& +2 \pi \sum_{i} D_{I_{i} S}^{\text {ori }} I_{z i} S_{z}+2 \pi \sum_{i, j(i<j)} J_{i j} \mathbf{I}_{\mathbf{i}} \cdot \mathbf{I}_{\mathbf{j}} \\
& +2 \pi \sum_{i} J_{I_{i} S} I_{z i} S_{z} .
\end{aligned}
$$

The equilibrium spectrum of the protons and the fluorine is given in Fig. 1. To use this spin system for QIP, one needs to identify the energy levels of the system and to assign each transition to a pair of these levels. There are two possible methods. One well-known method is to numerically diagonalize the Hamiltonian of the system, with some initially guessed parameters (chemical shifts, dipolar, and $J$-couplings) and iteratively fit the calculated and observed spectra [18]. The second method is the use of Z-COSY two-dimensional (2D) experiment which identifies connected transitions and draws automatically the energy-level diagram [26]. So far Z-COSY has been used only for homonuclear spins. This paper consists of two parts. In the first part (Section 2) we introduce a heteronuclear Z-COSY experiment and utilize it to obtain the complete energy level diagram of this dipolar coupled five spin system. In the second part (Section 3), we demonstrate that this spin system can be used as a 5-qubit system by preparing pseudopure states, performing $\mathrm{C}^{n}$-NOT operations and controlled SWAP operations, and creating and transferring entanglement using transition selective pulses.

\section{HET-Z-COSY}

The pulse sequence of a Z-COSY experiment is $90-t_{1}-\alpha-\tau-\beta-t_{2}$, where only the longitudinal magnetization is retained during the interval $\tau$ by either phase cycling or by a gradient pulse $[26,27]$. The $90^{\circ}$ pulse converts the equilibrium z-magnetization into coherences, which are frequency labeled during the period $t_{1}$. The small angle $\alpha$ pulse ensures that each cross-section parallel to $\omega_{2}$ from the resulting 2D spectrum is equivalent to a one-dimensional (1D) experiment in which the peak corresponding to the diagonal is selectively inverted. The directly connected transitions to the inverted transition are finally measured in the linear regime by a small angle $\beta$ pulse. The Z-COSY experiment has been successfully used to construct the energy level diagram in oriented homonuclear spin systems $[26,28]$. Here, the $\mathrm{Z}$-COSY experiment is extended to include a heteronucleus and we call this experiment as HET-Z-COSY.

For " $n$ " heteronuclear systems HET-Z-COSY experiment requires " $n$ " 2D experiments where each experiment observes one of the heteronucleus during $t_{2}$. The pulse sequence for HET-Z-COSY experiment is 

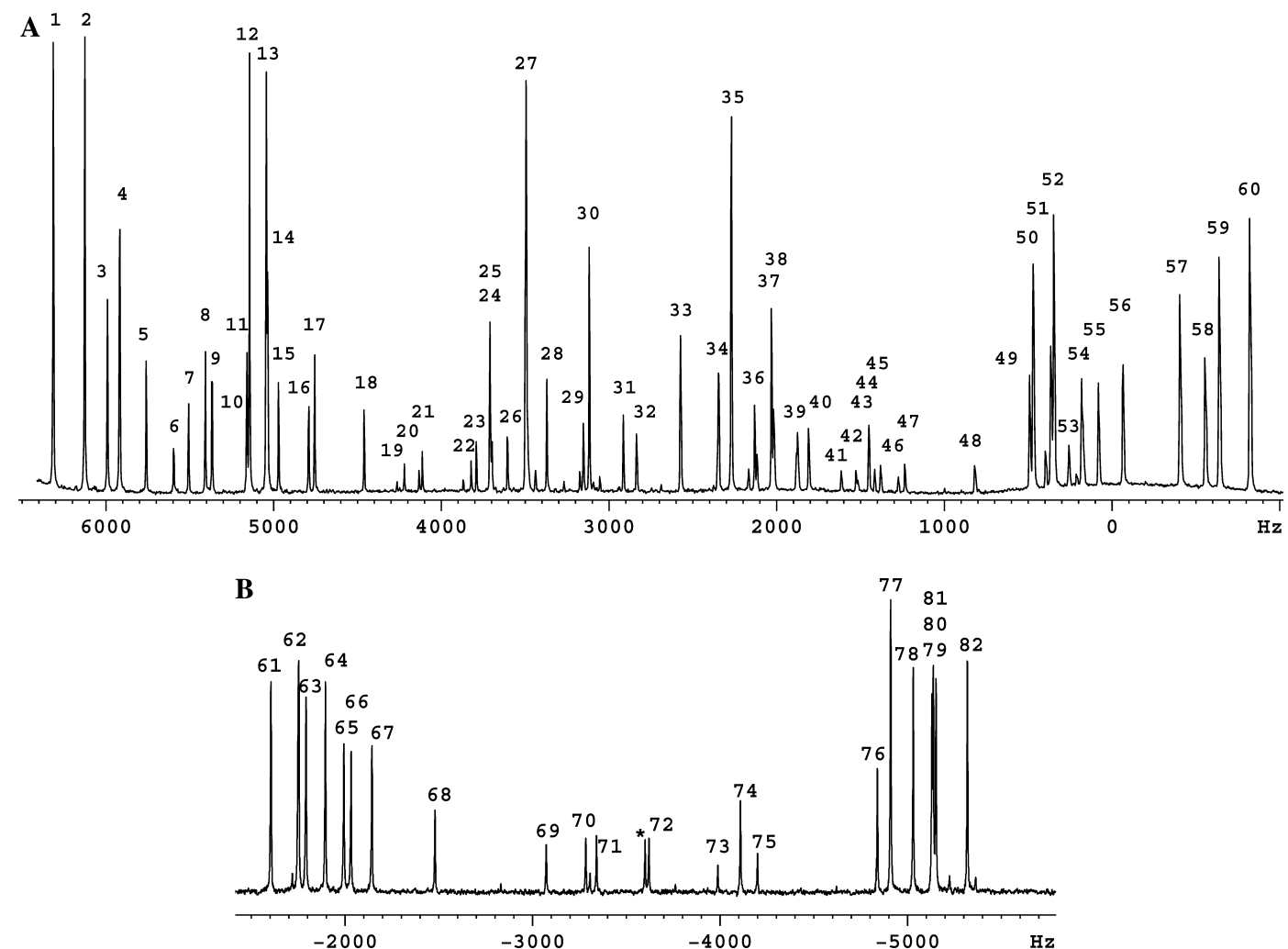

Fig. 1. Equilibrium spectrum of 1-chloro-2-fluorobenzene dissolved in liquid crystal solvent ZLI-1132. (A) The spectrum of four strongly coupled protons. (B) The fluorine spectrum. There are 82 transitions with significant intensities of which 1-60 are proton transitions and 61-82 are fluorine transitions. One fluorine transition did not show any connectivity in the subsequent HET-Z-COSY spectra and could not be assigned to any energy level in the energy-level diagram. This transition is shown by asterisk $(*)$.

$90-t_{1}-\alpha-\left(G z+k t_{1}\right)-\beta-t_{2}$, where the $90^{\circ}$ and $\alpha$ pulses are applied on all the nuclei, and $\beta$ is applied only on the observed nucleus. In the present case, two experiments have to be performed for detecting protons and fluorine, respectively. $G z$ is the gradient pulse which retains only the the longitudinal magnetization. Besides creating multi-spin zz terms [26], the $\alpha$-pulse creates all coherences including zero-quantum coherences. The gradient pulse destroys all other coherences as desired, except the homonuclear zero-quantum coherences. These zero-quantum coherences get partially converted into single quantum coherences by the last $\beta$ pulse causing the well-known zero-quantum interference in the $2 \mathrm{D}$ spectroscopy. To avoid this we have used the method of zero-quantum shifting [27] by incrementing the $\tau$ delay as $\left(G z+k t_{1}\right)$, where $k=2$ was used. This shifts the zero-quantum peaks in the $\omega_{1}$ dimension which are eventually removed by symmetrizing the spectrum [26].

The 2D spectrum of HET-Z-COSY on the systems of oriented 1-chloro-2-fluorobenzene obtained using $\alpha=\beta=10^{\circ}$, is shown in Fig. 2. The experimental spectrum when protons are observed in $t_{2}$, is given on the left side (Figs. 2A and $\mathrm{C}$ ), while the experimental spectrum when fluorine is observed is given on the right side (Figs. $2 \mathrm{~B}$ and $\mathrm{D})$. The spectrum in Fig. 2A has the diagonal and connectivity information between the proton transitions, while Fig. $2 \mathrm{C}$ has the connectivity information of the fluorine transitions to the proton transitions. Fig. 2D has the diagonal and connectivity information between the fluorine transitions, and Fig. 2B has the connectivity information of the proton transitions to the fluorine transitions. Every connectivity is mutual such that if transition ' $a$ ' is progressively connected to transition ' $b$,' then transition ' $b$ ' is also progressively connected to transition ' $a$.' Hence the cross peaks should be symmetric about the diagonal [26]. The symmetrized spectrum (free of zero-quantum and other artifacts) is given in Fig. 3. A connectivity matrix is constructed from the symmetric 2D spectrum, by taking cross-sections parallel to $\omega_{2}$ at each transition along $\omega_{1}$. From this connectivity matrix the complete energy-level diagram can be constructed following the method given in [26,28]. The complete energy level diagram constructed by the above procedure is given in Fig. 4. All the steps including construction of the juxtaposed 2D spectrum, symmetrization, construction of the connectivity matrix, and the construction of the complete energy-level diagram are automated by a program written in Matlab [29].

In this 5 -spin system, there are $2^{5}=32$ energy levels. The proton transitions connect energy levels within two separate domains (A and B) of 16 energy levels each, corresponding, respectively, to $+1 / 2$ and $-1 / 2$ states of 


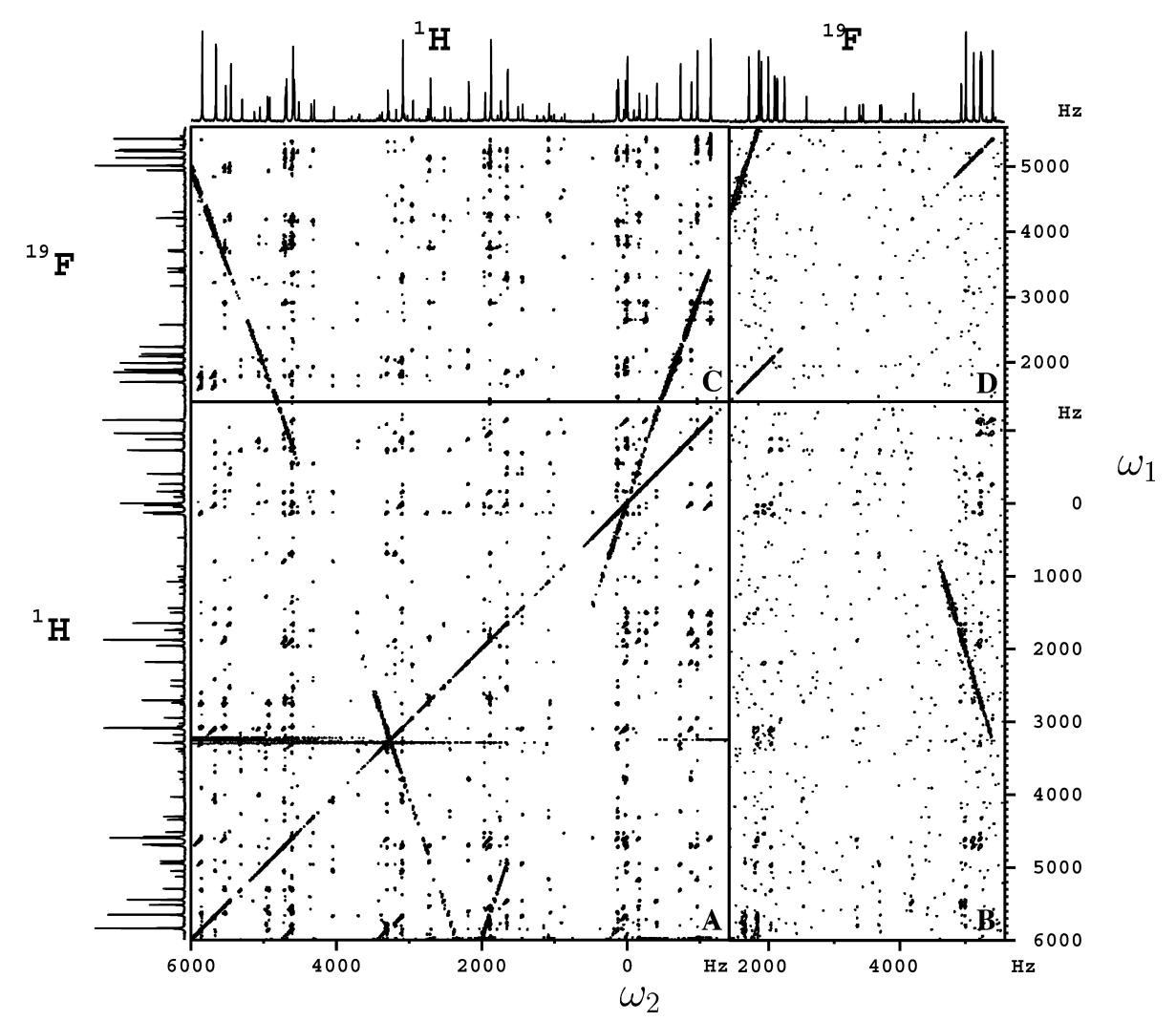

Fig. 2. Phase-sensitive HET-Z-COSY spectrum of oriented 1-chloro-2-fluorobenzene. (A) and (C) is obtained when protons are observed in $t_{2}$ while (B) and (D) is obtained when fluorine is observed in $t_{2} .2 \mathrm{~K} \times 2 \mathrm{~K}$ data points were collected with eight scans per $t_{1}$ increment. The data was zero-filled to $4 \mathrm{~K} \times 4 \mathrm{~K}$ before Fourier transform. The spectrum in (A) has the diagonal and connectivity information between proton transitions, while that in (C) has the connectivity information of the fluorine transitions to proton transitions. (D) has the diagonal and connectivity information between fluorine transitions, and (B) has the connectivity information of the proton transitions to fluorine transitions. Artifacts due to incomplete suppression of axial and double quantum diagonal are also visible in the spectrum. These artifacts are removed on symmetrization (Fig. 3).

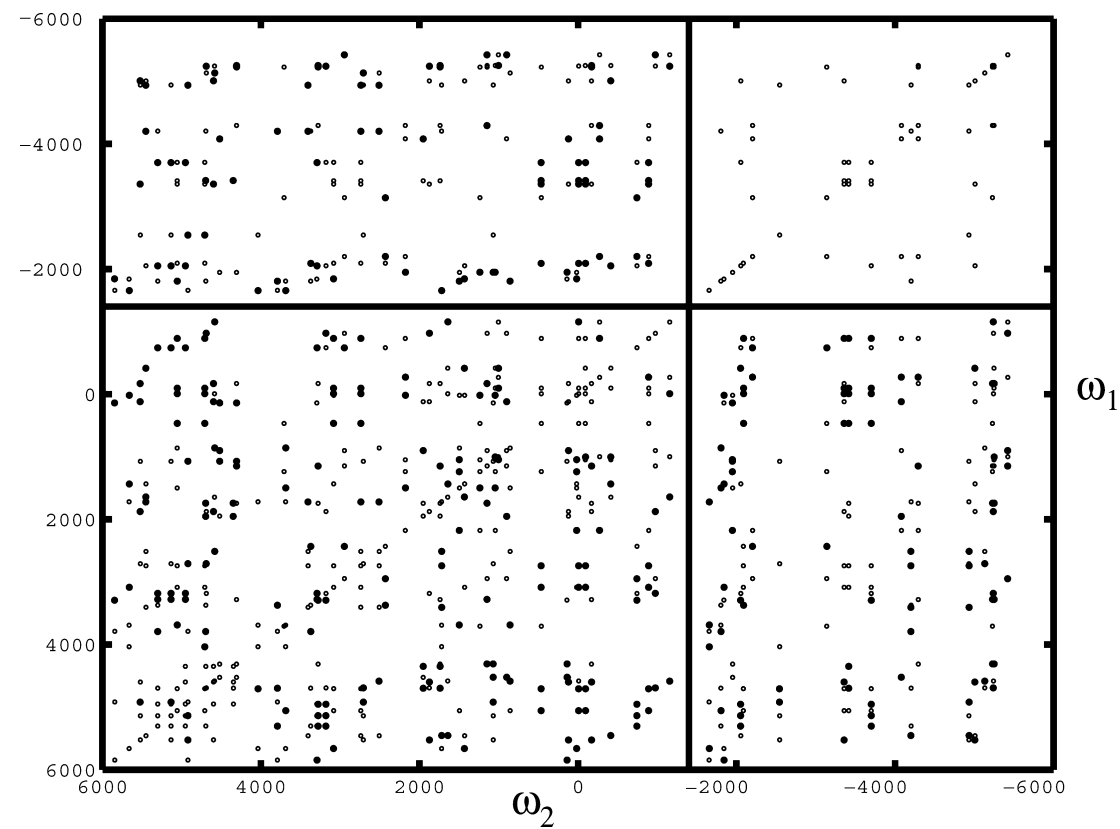

Fig. 3. Symmetric 2D spectrum of Fig. 2. A peak picking was performed on the 2D spectra of Fig. 2. The data from the two experiments were added to create a single $2 \mathrm{D}$ spectrum. This $2 \mathrm{D}$ spectrum was then symmetrized by a program written in Matlab [29]. The symmetrization is performed by retaining the lower absolute value of $\left(\omega_{i}, \omega_{j}\right)$, and $\left(\omega_{j}, \omega_{i}\right)$ cross-peaks, at both the cross-peak positions. For better visibility, the symmetric $2 \mathrm{D}$ spectrum is plotted with identical absolute intensity for all the peaks. The positive peaks are shown by filled circles while the negative peaks are shown by empty circles. 

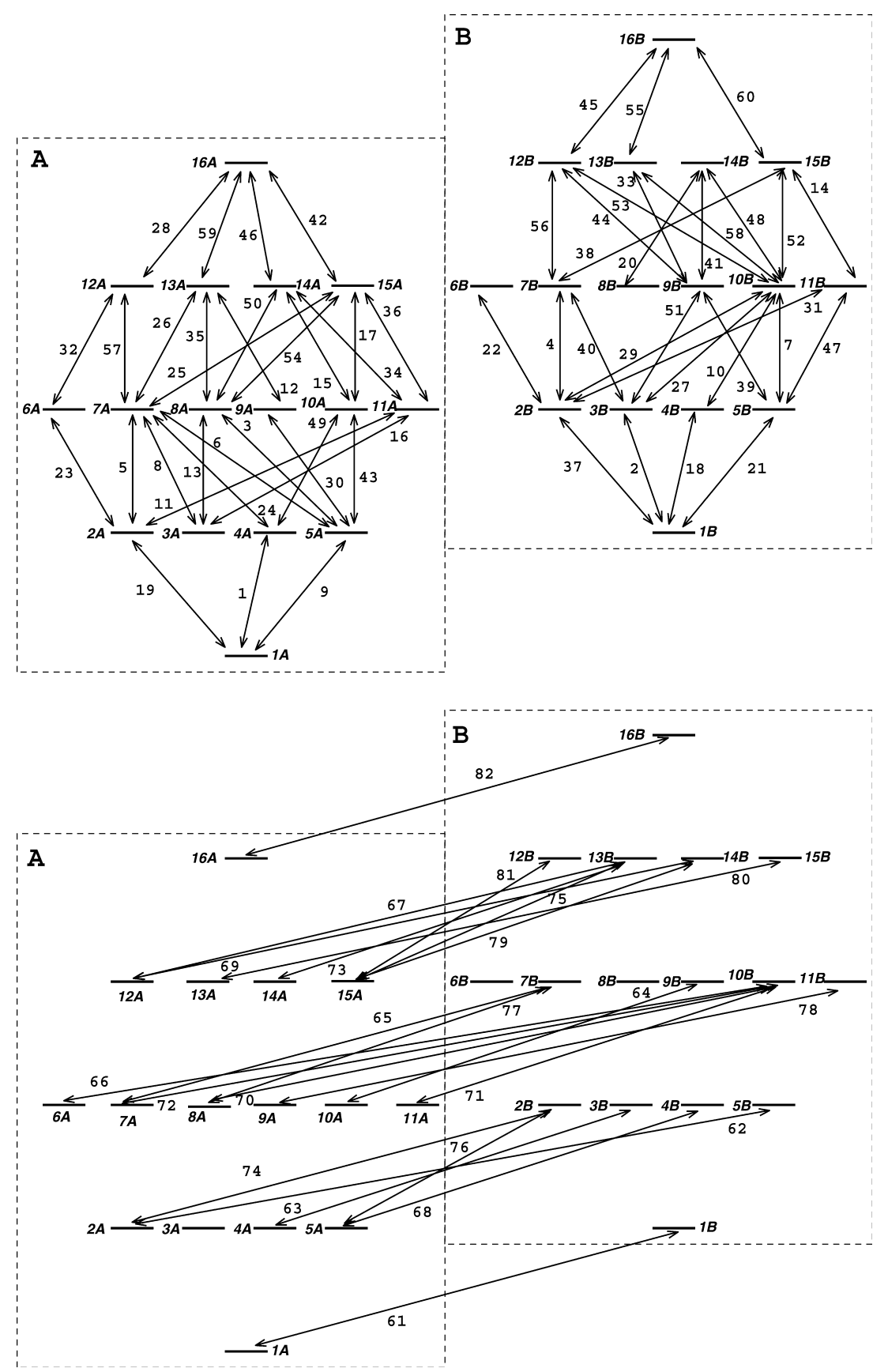

Fig. 4. Energy-level diagram of the five spin system of oriented 1-chloro-2-fluorobenzene. There are $32\left(2^{5}\right)$ energy levels which can be divided into two separate domains, A and B of 16 energy levels each. Domain A contains energy levels with fluorine in $+1 / 2$ state and domain B contains energy levels with fluorine in $-1 / 2$ state. Proton transitions connect energy levels within the two domains (upper figure) whereas fluorine transitions connect energy levels between A and B (lower figure).

the fluorine spin (Fig. 4). The fluorine transitions connect the levels from A domain to the B domain. The various transitions observed in the spectrum are given serial numbers (1-60 for proton and 61-82 for the fluorine transitions). Except the four extreme eigenstates all others are linear combinations of the product states of the four protons. The extreme eigenstates are 1A, 16A, $1 \mathrm{~B}$, and $16 \mathrm{~B}$, respectively, corresponding to the eigenstates $|\alpha \alpha \alpha \alpha \alpha\rangle,|\alpha \beta \beta \beta \beta\rangle,|\beta \alpha \alpha \alpha \alpha\rangle$, and $|\beta \beta \beta \beta \beta\rangle$, where the first state corresponds to the fluorine spin and last four to the protons.

\section{Quantum information processing using transition selective pulses}

Fig. 5 shows the labeling of the various eigenstates of the 5-spin system to 5-qubit labels. While labels can be 

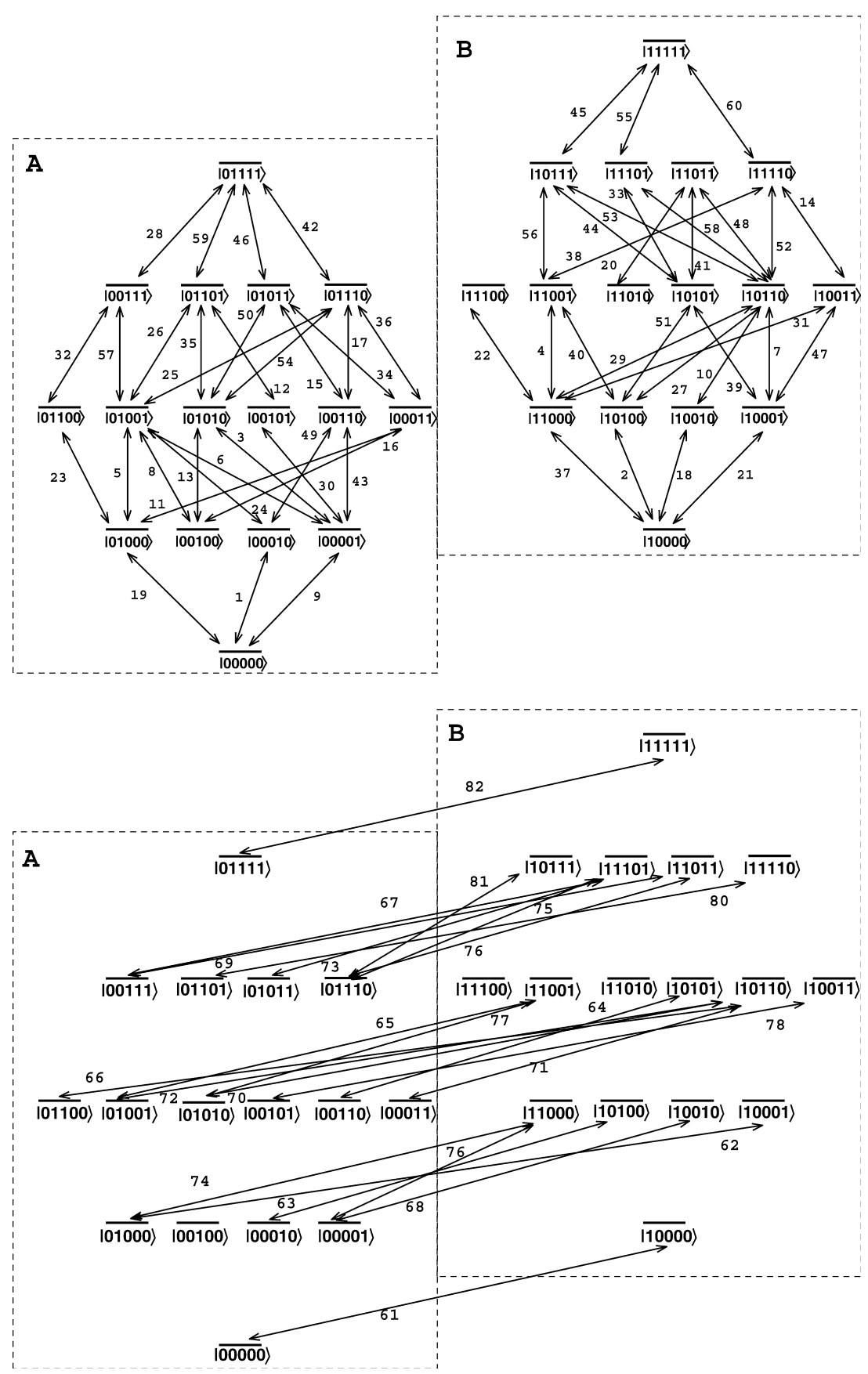

Fig. 5. Labeling of the eigenstates of 5-spin system to 5-qubit labels. The first qubit is in state $|0\rangle$ in domain A, whereas it is in state $|1\rangle$ in domain B. We have followed a conventional labeling scheme here such that in the limit of weak coupling, the state $\alpha(+1 / 2)$ is given label ' 0 ' and the state $\beta(-1 / 2)$ is given the label ' 1 .'

chosen for optimum execution of various logical operations, here we have retained a conventional labeling [30]. Transition selective $(\pi)$-pulse has a narrow bandwidth and when tuned to the resonance frequency of a specific transition in the spectrum, it exchanges the amplitudes of the two eigenstates which are connected by that transition [24]. Such pulses have already been used to simplify the implementation of several logical opera- tions for quantum information processing $[10,12,17$, 21,22,31-34].

\subsection{Preparation of pseudopure states}

Pseudopure states (PPS) are the starting point for implementation of various quantum algorithms by NMR. In a PPS, populations of all the states except 
one are equalized [16,17]. Among the various methods of creating PPS, we have implemented the method of pair of pseudopure states (POPS), originally suggested by Fung [21]. POPS requires two experiments: (i) equilibrium and (ii) a selective population inversion. Subtraction of these two population distributions yields zero populations for all the levels except the inverted pair, which in turn has equal positive/negative populations. This acts as a pair of pseudopure states. It has been demonstrated elsewhere, that for implementation of certain algorithms, POPS is sufficient to begin with [36]. Moreover, in an $N$-qubit system, POPS can also act as a $(N-1)$-qubit sub-system PPS.

Here, we have chosen to apply $(\pi)$ pulse on the fluorine transition 61 which connect $|00000\rangle \leftrightarrow|10000\rangle$. After the $(\pi)^{61}$ pulse, a gradient pulse is applied to kill any unwanted coherence created by the imperfection of the r.f. pulse. The final populations are measured by a small angle $(\pi / 20)$ proton and fluorine pulses, which convert within linear approximation, population differ-

A

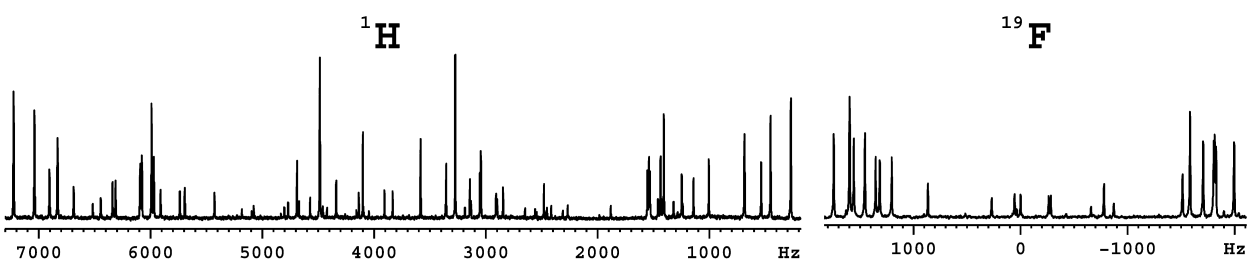

B
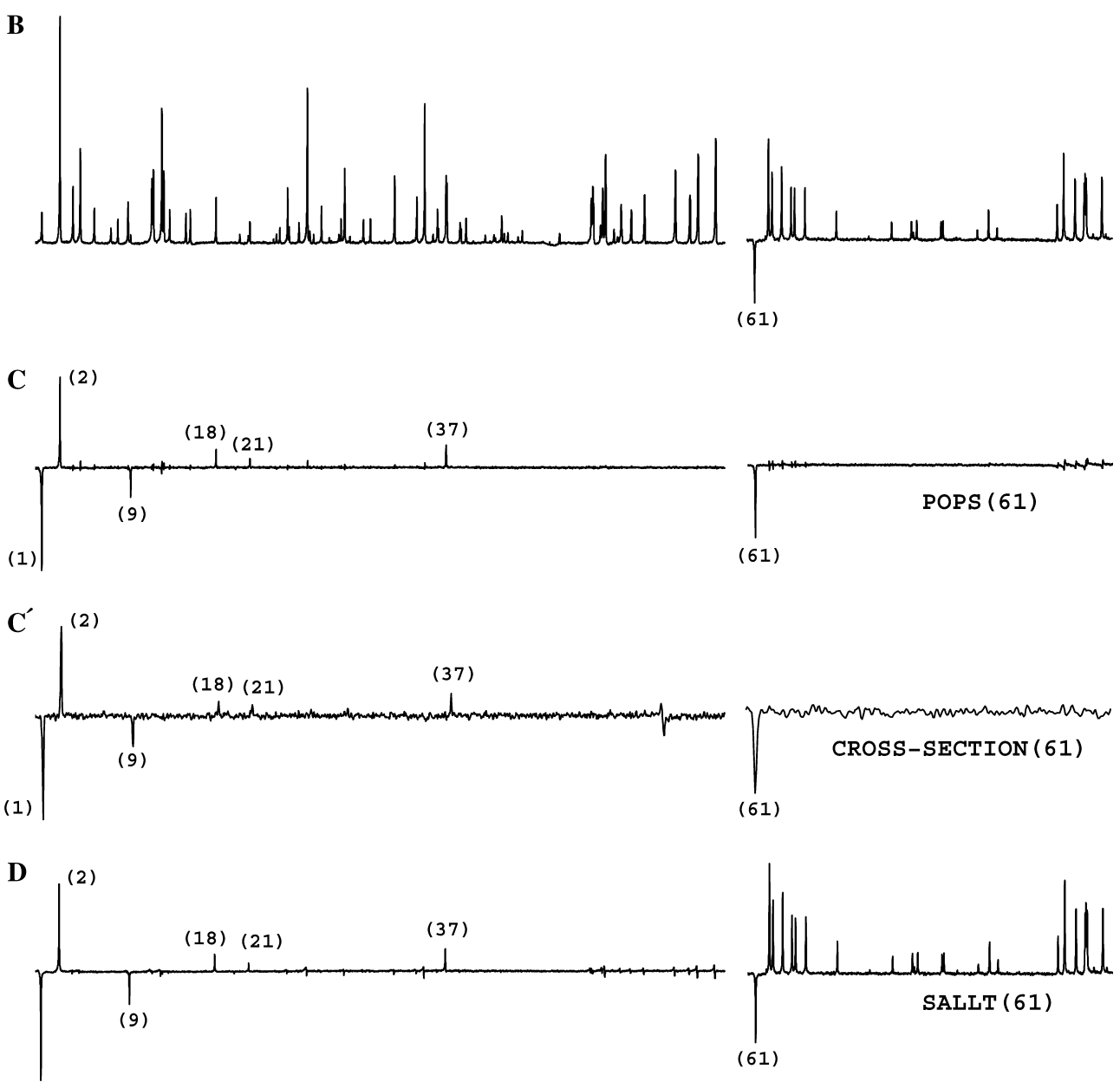

(1)

Fig. 6. Preparation of pseudopure states in the 5-qubit system of oriented 1-chloro-2-fluorobenzene. All spectra were obtained by using a readout small-angle $(\pi / 20)$ proton or fluorine pulse. (A) Equilibrium ${ }^{1} \mathrm{H}$ and ${ }^{19} \mathrm{~F}$ spectra. (B) Spectra corresponding to inversion of population between the states $|00000\rangle$ and $|10000\rangle$ implemented by a selective $(\pi)$ on transition 61. (C) Spectra obtained by subtraction of (A) from (B) yielding POPS(61) of $|10000\rangle\langle 10000|-| 00000\rangle\langle 00000|$. Note that the transitions $2,18,21$, and 37 are of opposite sign to that of 61 , confirming that they are progressively connected to 61 (see Fig. 5). The transitions 1 and 9 are of the same sign implying that they are, regressively, connected to 61 . Transition 19 is of very small intensity and not visible in (D). (C $\left.C^{\prime}\right)$ Cross-section parallel to $\omega_{2}$ of the spectrum in Fig. 2 at the $\omega_{1}$-frequency of transition 61 . (D) Spectra corresponding to the 4-qubit subsystem pseudopure state by SALLT (see text for details). 
ences into observable single quantum intensities. The resulting spectrum is given in Fig. 6B. Subtraction of equilibrium populations (Fig. 6A) from that corresponding to Fig. 6B yields the pair of pseudopure states $|10000\rangle\langle 10000|-| 00000\rangle\langle 00000|$. The resulting spectrum is given in Fig. 6C. Note that the transitions 2, 18, 21, and 37 are of opposite sign to that of 61 , as they are progressively connected to 61 (see Fig. 5) and transitions 1 and 9 are of the same sign since that they are regressively connected to 61 . The spectrum obtained after POPS contains only transitions originating from the two levels having non-zero populations. This effectively gives the various transitions connected to these levels. The same information is also available in the cross-sections of HET-Z-COSY spectrum at the frequency of transition connecting the two levels. A cross-section parallel to $\omega_{2}$ at the $\omega_{1}$-frequency of transition 61 from spectrum of Fig. 2 is given in Fig. 6C' . A good match of relative intensities of various transitions within each spectrum of Figs. 6C and $\mathrm{C}^{\prime}$ confirms the creation of POPS in Fig. 6C. It may be noted that, while Fig. $6 \mathrm{C}$ is achieved with a selective inversion of transition 61 , Fig. $6 \mathrm{C}^{\prime}$ is a cross-section of a $2 \mathrm{D}$ spectrum obtained using non-selective low angle pulses assuming perturbation and observation in the linear regime.

Another method of creating sub-system pseudopure state is; spatially averaged logical labeling technique (SALLT) [31]. A hard ( $\pi / 2)$ pulse on the protons equalize the populations within each subsystem [A and B], with the value differing between the two subsystems. A gradient pulses is subsequently applied to kill the coherences created in the process. A transition selective pulse $(\pi)$ on one of the fluorine transitions (we have chosen transition 61) then creates subsystem pseudopure states. The populations corresponding to this state are measured by a small angle $(\pi / 20)$ pulse and the resulting spectrum, is given in Fig. 6D. The proton spectra of Figs. 6C and D are nearly identical, confirming the creation of subsystem PPS. The populations of POPS and SALLT differ in the sense that POPS yields zero populations of all levels except one in each subsystem, while SALLT has equal population of all levels (not necessarily zero) except one in each subsystem. The fluorine spectrum in Fig. 6D is therefore identical to the spectrum in Fig. 6B.

\subsection{Implementation of controlled-NOT and controlled- SWAP gates}

Controlled-NOT gates are essential parts of any computation. A C $\mathrm{C}^{4}$-NOT gate will change the state of fifth qubit when the other four qubits are in the state $|1\rangle$. Proton transition 60 connects the states $|11110\rangle$ and |11111 (see Fig. 5). Hence a $(\pi)^{60}$ pulse performs $C^{4}$-NOT operation. We start from equilibrium state and implement the $\mathrm{C}^{4}$-NOT gate. A subsequent gradient pulse kills any coherences created by imperfection of the r.f. pulse. The output of the gate is stored in the final populations. The final populations, measured by small-angle $(\pi / 20)$ proton and fluorine pulses after implementing $\mathrm{C}^{4}$-NOT gate corresponding to $|11111\rangle \leftrightarrow|11110\rangle$, yield the spectrum given in Fig. 7B. Another POPS can be created by subtraction of this population distribution and the equilibrium populations. Subtraction of Fig. 7A from Fig. $7 \mathrm{~B}$ yields the POPS of $|11111\rangle\langle 11111|-| 11110\rangle\langle 11110|$. The corresponding spectrum is given in Fig. 7C. Creation of the POPS can also be confirmed by cross-sections parallel to $\omega_{2}$ of Fig. 2 at the $\omega_{1}$-frequency of the transition 60 , given in Fig. $7 \mathrm{C}^{\prime}$.

Controlled SWAP gates are becoming increasingly popular in quantum information processing [37,38]. A $\mathrm{C}^{3}$-SWAP gate swaps the state of last two qubits when the first three qubits are in the state $|1\rangle$. Essentially, this gate interchanges the amplitudes between $|11110\rangle$ and $|11101\rangle$. A sequence of three pulses $(\pi)^{58}-(\pi)^{52}-(\pi)^{58}$ achieves this gate. We start from the POPS of $|11111\rangle\langle 11111|-| 11101\rangle\langle 11101|$ prepared by subtraction of the equilibrium populations from the populations obtained by inversion of the proton transition 55 (Fig. 7D). The creation of this POPS is confirmed by the cross-section of Fig. 2 at the frequency of 55, given in Fig. 7D'. The subsequent implementation of $\mathrm{C}^{3}$-SWAP gate $\left[(\pi)^{58}-(\pi)^{52}-(\pi)^{58}\right]$ interchanges the populations between $|11110\rangle$ and |11101〉. A gradient pulse is applied after each $(\pi)$-pulse to destroy any coherences created by imperfection of pulses. The final state is the POPS of $|11111\rangle\langle 11111|-| 11110\rangle\langle 11110|$, and the resulting proton and fluorine spectra measured by a small angle $(\pi /$ 20) pulses is given in Fig. 7E. A good match between Fig. 7E and Fig. 7C confirms the implementation of $\mathrm{C}^{3}$-SWAP gate.

\subsection{Entanglement creation and transfer}

Entanglement is a remarkable property of quantum systems, which has no classical analogue. Entanglement is also a key requirement for many quantum information processing protocols. Many of these protocols require transfer of entanglement between qubits. Entanglement transfer has become specially useful in the cases where one needs to transfer data to the quantum memory (qubits with less decoherence that are used for storing data) from the fast processor (qubits have decoherence but can perform universal logic operations) in a manner similar to the protocol proposed by Chuang et al. [39]. Entanglement transfer has been demonstrated previously by Cory and co-workers [40] in liquid state NMR of weakly coupled 4-qubit spin-1/2 system. Here, we demonstrate entanglement transfer in this strongly coupled system by using the last 4-qubits of the 5-qubit system with the first qubit acting as an ancillary bit. We start by preparing the 4-qubit subsystem pseudopure state by SALLT 
$\mathbf{A}$

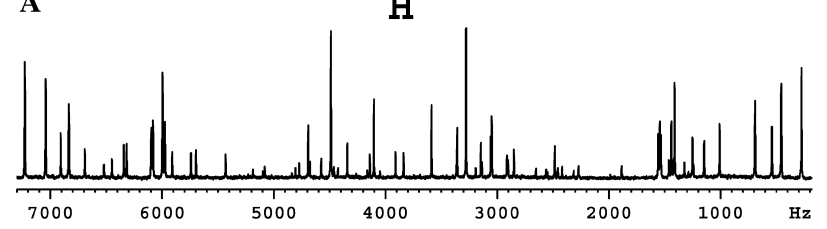

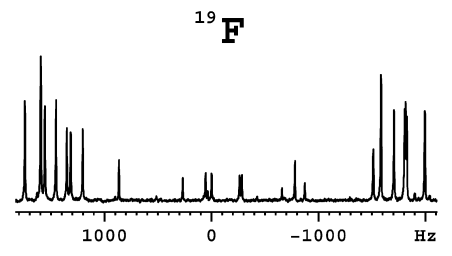

B

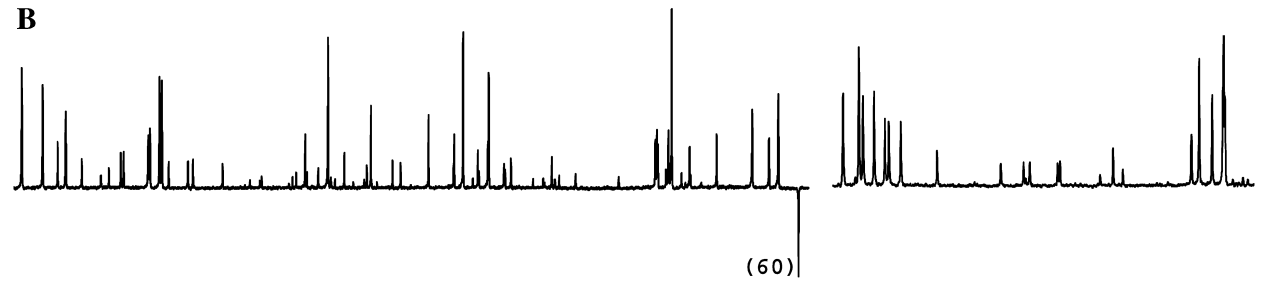

C
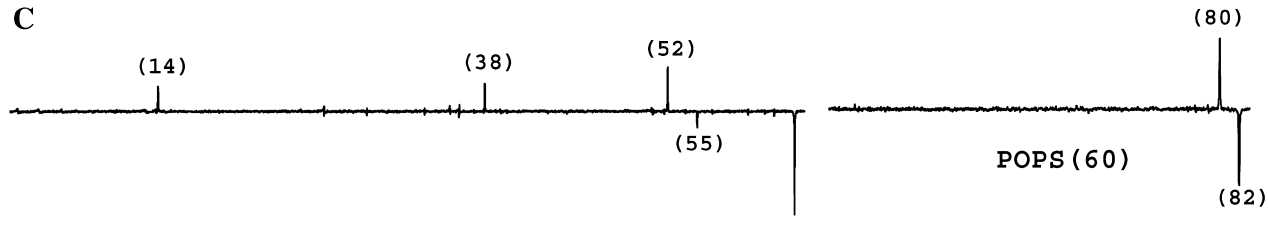

$\mathbf{C}^{\prime}$
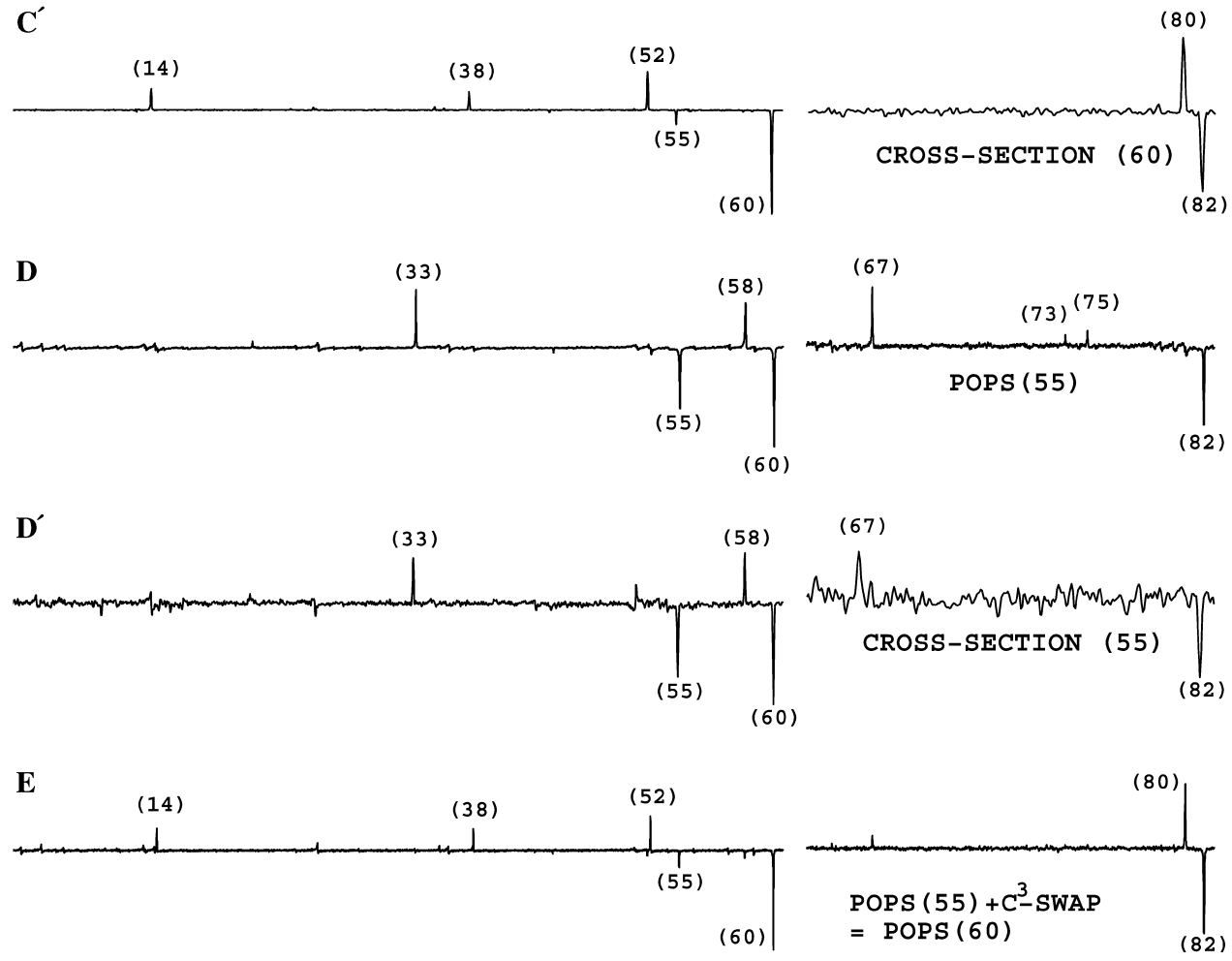

Fig. 7. Implementation of logic operations in 5-qubit system of oriented 1-chloro-2-fluorobenzene. (A) Equilibrium ${ }^{1} \mathrm{H}$ and ${ }^{19} \mathrm{~F}$ spectra. (B) Spectra corresponding to $\mathrm{C}^{4}$-NOT gate, implemented by a selective $(\pi)$ on transition 60 , which connects $|11110\rangle \leftrightarrow|1111\rangle$ (see Fig. 5). (C) Spectra corresponding to the creation of POPS(60) $|11111\rangle\langle 11111|-| 11110\rangle\langle 11110|$ by subtraction of (A) from (B). In these spectra, transitions 14, 38, 52, and 80 are of opposite sign to that of 60 , signifying that they are progressively connected to 60 , while 55 , and 82 are of the same sign confirming that they are regressively connected. The transition 45 is of very low intensity and not visible in $(\mathrm{C})$ and $\left(\mathrm{C}^{\prime}\right)$. $\left(\mathrm{C}^{\prime}\right)$ Cross-section parallel to $\omega_{2}$ of Fig. 2 at the $\omega_{1}$-frequency of transition 60. (D) POPS(55) of $|11111\rangle\langle 11111|-| 11101\rangle\langle 11101<|$ created by a selective $(\pi)$ pulse on 55 , and a subsequent subtraction of equilibrium. Transitions $33,58,67,73$, and 75 are of opposite sign to that of 55 , signifying that they are progressively connected to 55 (see Fig. 5). The transitions 60 and 82 are of the same sign confirming that they are regressively connected to 55 . Transition 45 is of very low intensity and not visible in (D). ( $\left.\mathrm{D}^{\prime}\right)$ Horizontal cross-section of Fig. 2 at the frequency of transition 55. (E) Implementation of $\mathrm{C}^{3}$-SWAP gate on the POPS of (D). A sequence of three selective pulses $(\pi)^{58}-(\pi)^{52}-(\pi)^{58}$ implements the $\mathrm{C}^{3}$-SWAP gate thereby creating the POPS of $|11111\rangle\langle 11111|-| 11110\rangle\langle 11110|$. A good match between (E) and (C) confirms implementation of $\mathrm{C}^{3}$-SWAP gate. 
(Fig. 6D). We chose to perform further operations in only one of the subsystems (where 1st qubit is in state |1 $\rangle$ ) [domain B]. Hence, in the next paragraph, only the states of the last four qubits are given, but they are to be understood as the states with first qubit being $|1\rangle$.
Starting from the $|0000\rangle$ pseudopure state, uniform superposition of 2 nd qubit was created by a $(\pi / 2)^{2}$ pulse: $|0000\rangle \rightarrow|0000\rangle+|0100\rangle$ (Fig. 5A and B domain). The states were tomographed using 2D Fourier transform technique [35]. Two-dimensional spectra for
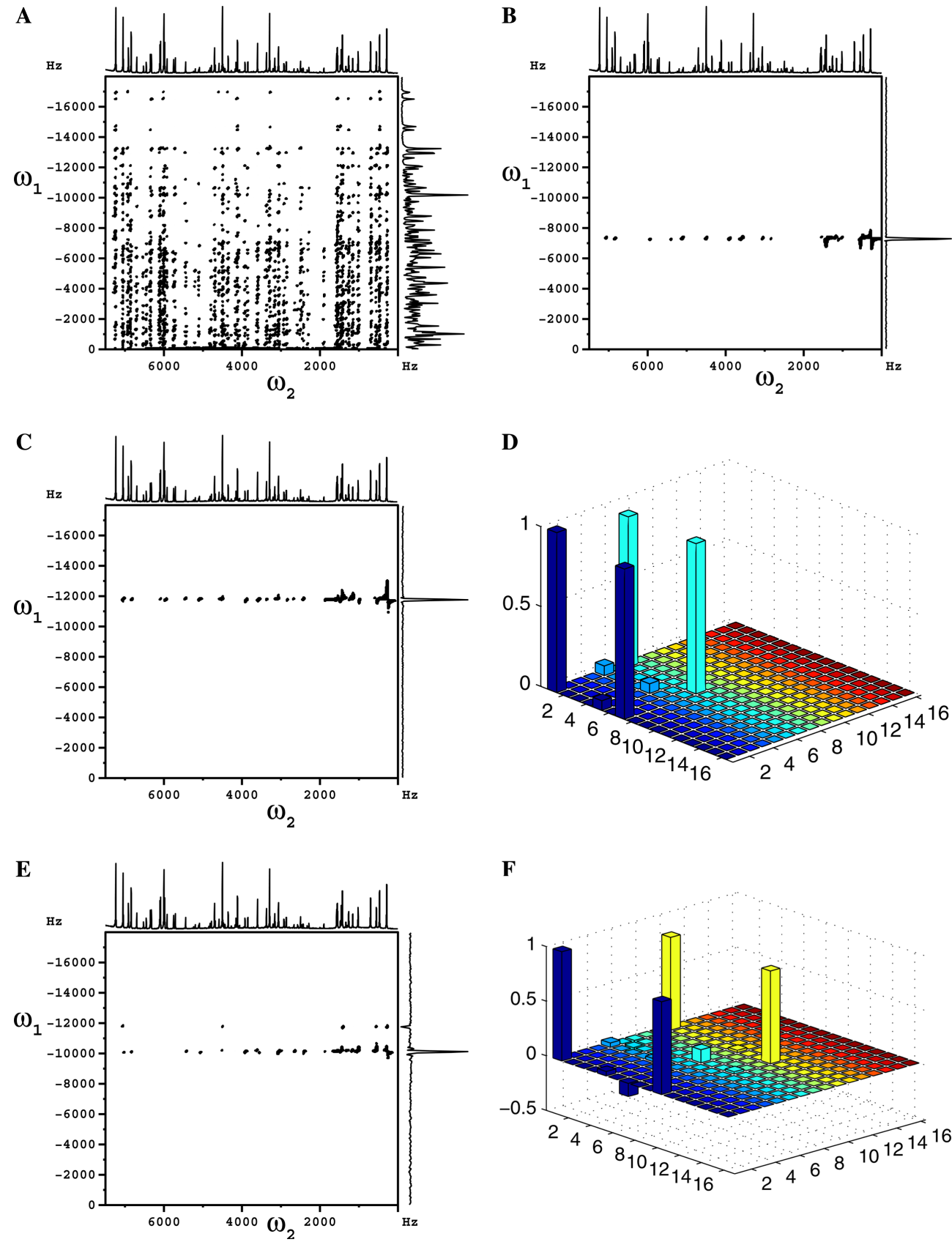

Fig. 8. Entanglement transfer within the last 4-qubits of the 5-qubit system of oriented 1-chloro-2-fluorobenzene. In all $2 \mathrm{D}$ experiments a date matrix of $1 \mathrm{~K} \times 1 \mathrm{~K}$ were collected all zero-filled to $2 \mathrm{~K} \times 2 \mathrm{~K}$ before Fourier transform. (A) Starting from equlibrium, multiple quantum spectrum of the four protons obtained by a pulse sequence of $(\pi / 2)-\tau-(\pi / 2)$, where $\tau=30 \mathrm{~ms}$, followed by the pulse sequence of tomography [35]. (B) Spectrum corresponding to measurement of all off-diagonal elements of the state $|0000\rangle+|0100\rangle$. A single coherence along $\omega_{1}$ at the frequency of transition 2 , $\left(\omega^{(2)}\right)$ and absence of any other coherences confirm creation of this state. (C) Spectrum corresponding to measurement of all off-diagonal elements of the entangled state $|0000\rangle+|0110\rangle$. A double quantum coherence along $\omega_{1}$ at the frequency $\omega^{(2)}+\omega^{(27)}$ and absence of any other coherences confirm creation of EPR coherences. (D) Corresponding tomographed density matrix using the method outlined in [35]. (E) Spectrum corresponding to measurement of all off-diagonal elements of the state after entanglement transfer. A double-quantum coherence is observed along $\omega_{1}$ at the frequency $\omega^{(2)}+\omega^{(40)}$ with a small coherence at $\omega^{(2)}+\omega^{(27)}$. (F) Corresponding tomographed density matrix confirm transfer of entanglement with $85 \%$ fidelity. 
measurement of all off-diagonal elements of the density matrix are given in Fig. 8. Fig. 8A contains the totality of the multiple quantum (MQ) spectrum of the four protons obtained by a $(\pi / 2)-\tau-(\pi / 2)$ pulse sequence starting from equilibrium population distribution. Fig. $8 \mathrm{~B}$ on the other hand contains the MQ spectrum starting from the state of $|0000\rangle+|0100\rangle$ and shows the presence of a single quantum coherence of this state at frequency of transition '2' $\left(\omega^{(2)}\right)$ along $\omega_{1}$. A controlled-NOT gate between 2 nd and 3 rd qubit is implemented by a $(\pi)^{27}$ pulse. This gate changes the state of third qubit when the second qubit is in state $|1\rangle$ and creates an entangled EPR (Einstein-Podolsky-Rosen [42]) pair between the 2nd and 3rd qubit given by: $(|0000\rangle+|0110\rangle)$. The presence of the double quantum coherence with frequency $\omega^{(2)}+\omega^{(27)}$ along $\omega_{1}$ in the $2 \mathrm{D}$ spectrum for tomography of all off-diagonal elements in Fig. 8C and the absence of all other coherences, indicates the creation of the EPR coherence. The tomographed density matrix of the B domain, given in Fig. 8D, confirm creation of the EPR state. A sequence of three transition selective pulses $(\pi)^{27}-(\pi)^{40}-(\pi)^{27}$ swaps the states of $|0110\rangle$ and $|1001\rangle$, thereby transferring the entanglement to the first and fourth qubit: $(|0000\rangle+|1001\rangle)$. Hence the entanglement initially created between the second and third qubit is now transfered to the first and fourth qubit, while the second and third qubit are in the $|00\rangle$ state and can be used for further computation. All the pulses and the receiver were phase cycled by a standard CYCLOPS phase cycle [24]. The presence of the double quantum coherence with frequency $\omega^{(2)}+\omega^{(40)}$ along $\omega_{1}$ in the 2D spectrum in Fig. 8E and the resulting tomograph of the $\mathrm{B}$ domain, given in Fig. $8 \mathrm{~F}$, confirm that the entanglement has been transferred with $85 \%$ fidelity.

\section{Conclusion}

Residual dipolar couplings can be used to increase the number of qubits for quantum information processing. We have used a molecule containing five spins oriented in a liquid crystal matrix, and exploited the residual dipolar coupling to demonstrate it as a 5-qubit quantum computer. Transition assignment is performed using HET-Z-COSY experiment and qubit addressability is achieved by transition selective pulses. It is expected that using this protocol higher qubits can be achieved. Preliminary experiments promise a 8-qubit system [41].

For the implementations reported in this paper, evolution under the internal Hamiltonian was not explored. It is however interesting to investigate how effectively the evolution under internal Hamiltonian can be manipulated to implement quantum algorithms in these systems. Efforts are ongoing in this direction in our laboratory, and recently a new meth- od of preparing pseudopure states in oriented systems by exciting selected multiple quantum using evolution under effective dipolar Hamiltonian, has been reported [43].

\section{Acknowledgments}

The authors thank T.S. Mahesh, N. Suryaprakash, and K.V. Ramanathan for useful discussions. The use of DRX-500 NMR spectrometer funded by the Department of Science and Technology, New Delhi, at the Sophisticated Instrument's Facility, Indian Institute of Science, Bangalore, is also gratefully acknowledged. AK acknowledges "DAE-BRNS" for the award of "Senior Scientists scheme," and DST for a research grant on "Quantum Computing using NMR techniques."

\section{References}

[1] R.P. Feynman, Simulating physics with computers, Int. J. Theor. Phys. 21 (1982) 467.

[2] D. Deutsch, R. Jozsa, Rapid solution of problems by quantum computation, Proc. R. Soc. Lond. A 400 (1985) 97.

[3] L.K. Grover, Quantum mechanics helps in searching for a needle in a haystack, Phys. Rev. Lett. 79 (1997) 325.

[4] P.W. Shor, Polynomial-time algorithms for prime factorization and discrete algorithms on quantum computer, SIAM Rev. 41 (1999) 303-332.

[5] J. Preskill, Lecture notes for Physics 229: Quantum information and computation, Available from: <http://theory.caltech.edu/ people/preskill/>.

[6] M.A. Nielsen, I.L. Chuang, Quantum Computation and Quantum Information, Cambridge University Press, Cambridge, 2000.

[7] D. Bouwmeester, A. Ekert, A. Zeilinger, The Physics of Quantum Information, Springer, Berlin, 2000

[8] I.L. Chuang, L.M.K. Vanderspyen, X. Zhou, D.W. Leung, S. Llyod, Experimental realization of a quantum algorithm, Nature (London) 393 (1998) 1443.

[9] J.A. Jones, M. Mosca, Implementation of a quantum algorithm on a nuclear magnetic resonance quantum computer, J. Chem. Phys. 109 (1998) 1648.

[10] N. Lindan, H. Barjat, R. Freeman, An implementation of Deutsch-Jozsa algorithm on a three-qubit NMR quantum computer, Chem. Phys. Lett. 296 (1998) 61.

[11] I.L. Chuang, N. Gershenfeld, M. Kubinec, Experimental implementation of fast quantum searching, Phys. Rev. Lett. 80 (1998) 3408-3411.

[12] Kavita Dorai, Arvind, Anil Kumar, Implementing quantumlogic operations, pseudopure states, and the Deutsch-Jozsa algorithm using noncommuting selective pulses in NMR, Phys. Rev. A. 61 (2000) 042306

[13] L.M.K. Vanderspyen, Matthias Steffen, Gregory Breyta, C.S. Yannoni, M.H. Sherwood, I.L. Chuang, Experimental realization of Shor's quantum factoring algorithm using nuclear magnetic resonance, Nature 414 (2001) 883.

[14] Ranabir Das, Anil Kumar, Use of quadrupolar nuclei for quantum-information processing by nuclear magnetic resonance: implementation of a quantum algorithm, Phys. Rev. A 68 (2003) 032304 . 
[15] D.G. Cory, A.F. Fahmy, T.F. Havel, Ensemble quantum computing by NMR spectroscopy, Proc. Natl. Acad. Sci. USA 94 (1997) 1634.

[16] N. Gershenfeld, I.L. Chuang, Bulk spin-resonance quantum computation, Science 275 (1997) 350.

[17] D.G. Cory, M.D. Price, T.F. Havel, Nuclear magnetic resonance spectroscopy: an experimentally accessible paradigm for quantum computing, Physica D 120 (1998) 82.

[18] P. Diehl, C.L. Khetrapal, NMR-Basic Principles and Progress, Springer-Verlag, New York, 1996, vol. 1, 1995.

[19] C.S. Yannoni, M.H. Sherwood, D.C. Miller, I.L. Chuang, L.M.K. Vandersypen, M.G. Kubinec, Nuclear magnetic resonance quantum computing using liquid crystal solvents, Appl. Phys. Lett. 75 (1999) 3563-3565.

[20] M. Marjanska, I.L. Chuang, M.G. Kubinec, Demonstration of quantum logic gates in liquid crystal nuclear magnetic resonance, J. Chem. Phys. 112 (2000) 5095.

[21] B.M. Fung, Using pair of pseudopure states for NMR quantum computing, Phys. Rev. A 63 (2001) 022304.

[22] B.M. Fung, Pairs of pseudopure states for 4- and 5-qubit nuclear magnetic resonance systems, J. Chem. Phys. 115 (2001) 8044.

[23] T.S. Mahesh, Neeraj Sinha, K.V. Ramanathan, Anil Kumar, Ensemble quantum-information processing by NMR: implementation of gates and creation of pseudopure states using dipolar coupled spins as qubits, Phys. Rev. A 66 (2002) 022313.

[24] R.R. Ersnt, G. Bodenhausen, A. Wokaun, Principles of Nuclear Magnetic Resonance in One and Two Dimensions, Oxford University Press, London, 1987.

[25] T.S. Mahesh, Neeraj Sinha, Arindam Ghosh, Ranabir Das, N. Suryaprakash, Malcolm H. Levitt, K.V. Ramanathan, Anil Kumar, Quantum information processing by NMR using strongly coupled spins, Current Science (85) (7) (2003) 932.

[26] R.C.R. Grace, Anil Kumar, Flip-angle dependence of nonequilibrium states yielding information on connectivity of transitions and energy levels of oriented molecules, J. Mag. Reson. 99 (1992) 81.

[27] M. Rance, G. Bodenhausen, G. Wagner, K. Wuthrich, R.R. Ernst, A systematic approach to the suppression of $J$ cross peaks in 2D exchange and 2D NOE spectroscopy, J. Mag. Reson. 62 (1985) 497.

[28] R.C.R. Grace, N. Suryaprakash, New Advances in Analytical Techniques, vol. 1, Atta-ur-Rahman, Harwood Academic Press, The Netherlands, 2000

[29] Rangeet Bhattacharyya, Private communication (program available on request via e-mail to rangeet@physics.iisc.ernet.in).

[30] Ranabir Das, Sukhendu Chakraborty, K. Rukmani, Anil Kumar Search for optimum labeling schemes in qubit systems for
Quantum Information processing by NMR Phys. Rev. A. (in press).

[31] T.S. Mahesh, Anil Kumar, Ensemble quantum-information processing by NMR: spatially averaged logical labeling technique for creating pseudopure states, Phys. Rev. A. 64 (2001) 012307.

[32] X. Peng, X. Zhu, X. Fang, M. Feng, K. Gao, M. Liu, Preparation of pseudopure states by line selective pulses in nuclear magnetic resonanace, Phys. Rev. A 65 (2002) 042315.

[33] T.S. Mahesh, Kavita Dorai, Arvind, Anil Kumar, Implementing logic gates and Deutsch-Jozsa quantum algorithm by two-dimensional NMR using spin-and transition-selective pulses, J. Magn. Reson. 148 (2001) 95.

[34] Ranabir Das, T.S. Mahesh, Anil Kumar, Implementation of conditional phase-shift gate for quantum information processing by NMR, using transition-selective pulses, J. Magn. Reson. 54 (2002) 46 .

[35] Ranabir Das, T.S. Mahesh, Anil Kumar, Efficient quantum state tomography for quantum information processing using twodimensional fourier transform technique, Phys. Rev. A. 67 (2003) 062304.

[36] Vladimir L. Ermakov, B.M. Fung, Nuclear magnetic resonance implementation of the Deutsch-Jozsa algorithm using different initial states, J. Chem. Phys. 118 (2003) 10376.

[37] J.A. Smolin, D.P. DiVincenzo, Five two-bit quantum gates are sufficient to implement the quantum Fredkin gate, Phys. Rev. A 53 (1996) 2855-2856.

[38] Christopher C. Gerry, R.A. Campos, Generation of maximally entangled photonic states with a quantum-optical Fredkin gate, Phys. Rev. A 64 (2001) 063814.

[39] D. Gottesman, I.L. Chuang, Demonstrating the viability of universal quantum computation using teleportation and singlequbit operations, Nature (Lond.) 402 (1999) 390

[40] N. Boulant, E.M. Fortunato, M.A. Pravia, G. Teklemariam, D.G. Cory, T.F. Havel, Entanglement transfer experiment in NMR quantum information processing, Phys. Rev. A 65 (2002) 024302 .

[41] Ranabir Das, Rangeet Bhattacharyya and Anil Kumar, In search of higher qubit systems for quantum information processing by NMR, presented in 10th National Magnatic Resonance Symposium, SINP, Kolkata, February 17-2 (2004).

[42] A. Einstein, B. Podolsky, N. Rosen, Can quantum-mechanical description of physical reality be considered complete, Phys. Rev. 47 (1935) 777.

[43] Jae-Seung Lee, A.K. Khitrin, Preparation of pseudopure state in a cluster of dipolar-coupled spins with "unresolved" spectrum, quant-ph/0402132. 